\title{
Internet das Coisas: a busca do conceito e as perspectivas futuras sobre sua
}

\section{aplicabilidade}

\author{
Internet of Things: the search for the concept and future perspectives on its applicability \\ Internet de Las Cosas: la búsqueda del concepto y perspectivas futuras sobre su aplicabilidad
}

Matheus Martins Santos

ORCID: https://orcid.org/0000-0002-8450-6994 Universidade Federal dos Vales Jequitinhonha e Mucuri, Brasil

E-mail: matheusms@gmail.com

Mauro Lúcio Franco

ORCID: https://orcid.org/0000-0003-2114-4399

Universidade Federal dos Vales Jequitinhonha e Mucuri, Brasil

E-mail: ml.franco@ufvjm.edu.br

Wederson Marcos Alves

ORCID: https://orcid.org/0000-0002-1105-1300

Universidade Federal dos Vales Jequitinhonha e Mucuri, Brasil

E-mail: wederson.alves@ufvjm.edu.br

Marcio Coutinho de Souza

ORCID: https://orcid.org/0000-0002-4238-1572

Universidade Federal dos Vales Jequitinhonha e Mucuri, Brasil

E-mail: marcio.souza@ufvjm.edu.br

Geórgia Fernandes Barros

ORCID: https://orcid.org/0000-0003-1362-9548 Universidade Federal dos Vales Jequitinhonha e Mucuri, Brasil E-mail: georgia.barros@ufvjm.edu.br

Magnum Moreira da Silva

ORCID: https://orcid.org/0000-0001-8797-1819 Universidade Federal dos Vales Jequitinhonha e Mucuri, Brasil E-mail: magnum.moreira@ufvjm.edu.br

\begin{abstract}
Resumo
Internet das Coisas é uma tradução da expressão Internet of Things (IoT), que nos remete a ideia de que a Internet pode estar presente em todas as coisas, expandindo a possibilidade de controle e coleta de dados. Esse artigo tem como objetivo buscar conceitos da IoT, descrevendo sua evolução, aplicabilidade em diversos segmentos, suas perspectivas futuras e seus maiores desafios para implementação em larga escala. Para tanto, se fez uso de pesquisa bibliográfica, analítica e descritiva em livros, artigos, dissertações, reportagens, documentos nacionais e internacionais. O artigo discute sobre a evolução da Internet, também retrata sobre IoT e a evolução das redes móveis de telefonia celular, traz algumas perspectivas futuras e demonstra alguns desafios para IoT. Ficou constatado que a IoT apesar dos desafios como a implementação da quinta geração das redes de telefonia, da segurança e privacidade dos dados gerados e transferidos, já é uma realidade com suas diversas aplicabilidades. A evolução dos dispositivos dos mais variados tipos integrados em smartphones, smart tvs, smartwatches, expandindo ainda mais o acesso à Internet no período hodierno, nos fornece informações que auxiliam nas tomadas de decisões. É fundamental não só a utilização destes dispositivos, mas também que todos conheçam seus conceitos, evolução, aplicabilidades e desafios. Sugere-se como proposta de pesquisas futuras temáticas envolvendo a inserção das pessoas em ambientes que não usem a IoT e ambientes nos quais as decisões são tomadas com o auxílio da IoT, por exemplo pesquisas com grupos de controle.
\end{abstract}

Palavras-chave: Tecnologia; Internet; Internet das Coisas; IoT.

\begin{abstract}
Internet of Things is a translation of the expression Internet of Things (IoT), which reminds us that the Internet can be present in all things, expanding the possibility of control and data collection. This article aims to search for IoT concepts, describing its evolution, applicability in various segments, its future perspectives and its greatest challenges for large-scale implementation. Therefore, bibliographic, analytical and descriptive research was used in books, articles, dissertations, reports, national and international documents. The article discusses the evolution of the Internet, also portrays IoT and the evolution of mobile cellular networks, brings some future perspectives and demonstrates some challenges for IoT. It was found that the IoT, despite the challenges such as the implementation of the fifth generation of telephone networks, the security and privacy of the data generated and transferred, is already a reality with its various applicability. The evolution of devices of the most varied types integrated into smartphones, smart tvs,
\end{abstract}


smartwatches, further expanding Internet access in today's period, provides us with information that helps in decisionmaking. It is essential not only to use these devices, but also that everyone knows their concepts, evolution, applicability and challenges. It is suggested as a proposal for future thematic research involving the insertion of people in environments that do not use IoT and environments in which decisions are taken with the help of IoT, for example, research with control groups.

Keywords: Technology; Internet; Internet of Things; IoT.

\section{Resumen}

Internet of Things es una traducción de la expresión Internet of Things (IoT), que nos recuerda que Internet puede estar presente en todas las cosas, ampliando la posibilidad de control y recopilación de datos. Este artículo tiene como objetivo buscar conceptos de IoT, describiendo su evolución, aplicabilidad en varios segmentos, sus perspectivas de futuro y sus mayores desafíos para la implementación a gran escala. Por lo tanto, se utilizó la investigación bibliográfica, analítica y descriptiva en libros, artículos, disertaciones, informes, documentos nacionales e internacionales. El artículo analiza la evolución de Internet, también describe la IoT y la evolución de las redes celulares móviles, aporta algunas perspectivas de futuro y demuestra algunos desafíos para la IoT. Se encontró que el IoT, a pesar de los desafíos como la implementación de la quinta generación de redes telefónicas, la seguridad y privacidad de los datos generados y transferidos, ya es una realidad con sus variadas aplicabilidades. La evolución de los dispositivos de los más variados tipos integrados en smartphones, smart tvs, smartwatches, ampliando aún más el acceso a Internet en el período actual, nos proporciona información que ayuda en la toma de decisiones. Es fundamental no solo utilizar estos dispositivos, sino también que todo el mundo conozca sus conceptos, evolución, aplicabilidad y retos. Se sugiere como propuesta para futuras investigaciones temáticas que involucren la inserción de personas en entornos que no utilizan IoT y entornos en los que se toman decisiones con ayuda de IoT, por ejemplo, investigación con grupos de control.

Palabras clave: Tecnología; Internet; Internet de las Cosas; IoT.

\section{Introdução}

A evolução da Internet promove cada vez mais a fusão do mundo real e virtual facilitando o nosso cotidiano. Particularmente, a Internet das Coisas nos proporciona um maior controle e obtenção de dados dos dispositivos conectados, que nos auxiliarão nas tomadas de decisões, benefícios estes que não consegue-se sem a interligação destes dispositivos na Internet, algo comum em sistemas legados como sistemas de monitoramento local, sensores offline e outros dispositivos tradicionais (Silva, \& et al., 2020).

Internet das Coisas é uma tradução da expressão Internet of Things (IoT), que nos remete a ideia de que a Internet pode estar presente em todas as coisas. Estes dispositivos conectados à Internet nos permitirão controlá-los remotamente e também transformar estes dispositivos em provedores de serviços, agregando a estes objetos comuns novas habilidades que irão automatizar tarefas, coleta de dados, criação de rotinas precisas e com direcionamento otimizado, gerando novas oportunidades.

Destaca-se que a IoT cada vez mais tem atualizado o conceito de redes de computadores. Para Tanenbaum (2011, p. 1) "redes de computadores é um conjunto de computadores autônomos interconectados por uma única tecnologia". Já Peterson \& Davie (2011) define que as redes de computadores são generalistas e os dados que trafegam não são específicos como toque de teclas, vídeo e voz e que se conectam somente dispositivos com finalidades especiais como terminais telefônicos e televisão. O termo "redes de computadores” já está obsoleto para Kurose \& Ross (2012), devido a diversos tipos diferentes de equipamentos e tecnologias não tradicionais que estão conectados na Internet (Santos, \& et al., 2016).

Apesar de fazer parte do nosso cotidiano nem todos tem conhecimento do termo IoT e é cada vez mais comum o conceito de Plug and Play, onde adquire-se o dispositivo seja computador, smart tv, smartphone, conecta-os na Internet e estão prontos para serem utilizados, sem saber que está conectado a IoT este usuário não tem o conhecimento da evolução da IoT, conceitos, diversas aplicabilidades e desafios enfrentados para o funcionamento ideal. Diante desses fatos surge o seguinte problema de pesquisa: Como identificar o que de fato é a IoT e quais os desafios e perspectivas futuras a serem identificadas para a implementação desta tecnologia em larga escala?

Por esta percepção, o presente trabalho tem como objetivo buscar conceitos da IoT, descrevendo sua evolução, 
aplicabilidade em diversos segmentos, suas perspectivas futuras e seus maiores desafios para implementação em larga escala.

O presente artigo está estruturado nas seguintes seções: introdução, problema e objetivos da pesquisa, referencial teórico, metodologia, considerações finais e referências.

\section{Metodologia}

Este artigo é classificado como uma pesquisa bibliográfica e descritiva. Como suporte metodológico foram utilizados os autores Gil (2017), Marconi (2017) e os autores que tratam da temática sobre IoT, redes de comunicação e tecnologia.

É classificada como bibliográfica pois sua fundamentação teórica bem como a identificação do estágio atual do conhecimento referente ao tema foi baseada em materiais já publicados como livros, dissertações, revistas, manuais etc (Marconi, 2017). Recorreu-se também a pesquisa descritiva com abordagem qualitativa com o objetivo de descrever grupos ou fenômenos como a evolução da tecnologia e identificar possíveis relações entre variáveis, os efeitos da tecnologia no nosso cotidiano (Gil, 2017).

Para construção deste artigo foi realizado a coleta e análise textual de livros, artigos, dissertações, reportagens, documentos nacionais e internacionais com as temáticas redes de computadores, Internet, IoT, telefonia móvel e segurança e privacidade.

\section{Referencial Teórico}

$\mathrm{Na}$ expectativa de buscar embasamento sobre a temática, nesta seção será discutido sobre a evolução da Internet contando o histórico que remonta o surgimento da Internet até os dias atuais; Internet das coisas e a evolução das redes móveis de telefonia celular contando conceito da Internet das coisas e a evolução das redes de telefonia móvel da primeira a quinta geração; Perspectivas futuras de aplicabilidades e desafios para Internet das coisas.

\subsection{A evolução da internet}

A Internet surgiu da necessidade de uma comunicação segura e tolerante a falhas, pois o Departamento de Defesa dos Estados Unidos no auge da guerra fria utilizava como meio de comunicação a rede de telefonia pública, considerada vulnerável pois todas as comunicações passavam por centrais de comutações telefônicas que eram estruturadas hierarquicamente e a destruição de poucas centrais estratégicas comprometeriam o funcionamento de todo o sistema de comunicação. Foi criada então uma organização centralizada de pesquisa de defesa a Advanced Research Projects Agency (ARPA) (Tanenbaum, 2011).

A ARPA como uma organização nova e com orçamento pequeno não possuía cientistas e laboratórios, trabalhava ofertando concessões e contratos a universidades e pessoas com ideias promissoras, como a de Paul Baran que sugeriu a criação de uma sub-rede comutada por pacotes, para atender a necessidade de acesso remoto a computadores. A construção desse projeto de sub-rede mais tarde ficou conhecido como Advanced Research Projects Agency Network (ARPANET) (Tanenbaum, 2011).

Em dezembro de 1969, a ARPANET entra em operação, interligando os nós: Universidade da Califórnia em Los Angeles, o Instituto de Pesquisa Stanford, a Universidade da Califórnia em Santa Bárbara e a Universidade de Utah, nós estes escolhidos por possuírem grande número de contratos com a ARPA e também por possuir configurações totalmente diferentes e incompatíveis aumentando o desafio técnico para implementação (Tanenbaum, 2011).

Ao final da década de 1970 a National Science Foundation (NSF) notou o impacto da ARPANET, que permitia o compartilhamento de informações dos cientistas de todo país e fomentou o projeto Computer Science Network (CSNET), que inicialmente conectava através de linhas discadas e privadas outras instituições a ARPANET e depois evoluiu para a criação 
da National Science Foundation Network (NSFNET), uma rede que expandiu rapidamente despertando o interesse de participação das organizações comerciais, deixando claro que o governo não podia continuar financiando a rede para sempre e este foi o primeiro processo em direção a comercialização (Tanenbaum, 2011).

Desde a criação dessas redes que precederam a Internet surgiram desafios como capacidade de conexões, velocidades maiores e novos meios de acesso, que são superados com pesquisas que resultam em novos tipos de tecnologias, meios de acesso e protocolos, como Transmission Control Protocol/Internet Protocol (TCP/IP), o Domain Name System (DNS), meios de transmissão dos dados como fibra óptica, comunicação sem fio e a World Wide Web (WWW ou Web) onde é acessado documentos hipermídia (Carvalho, 1997).

Internet é a interligação de várias redes de computadores ao redor do mundo. Tanenbaum (2011, p. 33) relata que a Internet "não é de modo algum uma rede, mas sim um vasto conjunto de redes diferentes que utilizam certos protocolos comuns e fornecem determinados serviços comuns" e (Brasil, 2014, sp) pondera Internet como "O sistema constituído do conjunto de protocolos lógicos, estruturado em escala mundial para uso público e irrestrito, com a finalidade de possibilitar a comunicação de dados entre terminais por meio de diferentes redes". O protocolo TCP/IP é o responsável por fornecer um serviço confiável e estabelecer um conjunto de regras para o envio e recebimento de dados entre dispositivos em diferentes redes (Carvalho, 1997).

Um dispositivo conectado à Internet utilizando o protocolo IP obrigatoriamente deve possuir um endereço que permite a identificação de origem e destino de um pacote. Um recurso que define o protocolo IP na versão 4 são seus endereços de 32 bits que permite $2^{32}$ endereços 4.294.967.296 (pouco mais de 4 bilhões) de dispositivos conectados simultaneamente. Quantidade suficiente para suportar a quantidade de dispositivos em sua época de criação, quando o acesso a recursos computacionais e a Internet eram restritos (Tanenbaum, 2011).

Com a evolução da Tecnologia e democratização do acesso à Internet o número de dispositivos conectados à Internet extrapolou a quantidade de endereços disponíveis, visando solucionar este problema foram desenvolvidos estratégias como Network Address Translation (NAT) e o Classless Inter-Domain Routing (CIDR) que permitem o compartilhamento e uma nova divisão de endereços IPs dão até hoje uma sobrevida ao IP versão 4. Este problema disponibilidade de endereços só será solucionado com a implementação protocolo IP versão 6 que permite 12834028237 e+38 (cerca de 340 undecilhões endereços), suficientes para suportar a evolução onde antes tinha-se somente computadores conectados para fins de pesquisa e agora tem-se outra realidade com computadores, smart tvs e smartphones, eletrodomésticos, assistentes virtuais, como podemos ver na figura 1.1 tornando uma rede cada vez mais heterogênea em tipos de dispositivos e objetivos de acesso (Tanenbaum, 2011).

As primeiras conexões internacionais entre Brasil e Exterior tiveram início em 1989, com o objetivo de permitir um compartilhamento mais rápido de informações entre os pesquisadores brasileiros e do exterior e eram conexões de ponto a ponto que interligavam a já existente Academic Network at São Paulo (ANSP), - rede que era mantida pela Fundação de Amparo à Pesquisa do Estado de São Paulo (Fapesp) e que interligava as principais instituições de ensino paulistas - ao laboratório de física de partículas em Chicago nos Estados Unidos, o Fermi National Accelerator Laboratory (Fermilab) e a outra conexão era entre o Laboratório Nacional de Computação Científica (LNCC) e a Universidade de Maryland, nos Estados Unidos (Agência Brasil, 2021).

Apesar de estarem conectados através das instituições americanas a comunicação entre os institutos brasileiros não eram realizadas diretamente, pois as redes utilizavam protocolos diferentes, uma informação requisitada por uma universidade de São Paulo ao LNCC obrigatoriamente deveria seguir até os Estados Unidos e voltar, aumentando significativamente o tempo de resposta. Este problema só foi solucionado em janeiro de 1991 com a normatização do TCP/IP nas redes, surgindo sim agora a Internet no Brasil (Oliveira, 2011). 
Em 1994 a então Empresa Brasileira de Telecomunicações (Embratel) disponibiliza a Internet comercialmente para cinco mil usuários testarem o novo serviço, expandindo a conexão que antes eram restritos a órgãos governamentais e instituições de pesquisa (Embratel, 2020). Em 1995 é criado o Comitê Gestor da Internet no Brasil (CGI.br), cujo a atribuição é estabelecer diretrizes estratégicas relacionadas ao uso e desenvolvimento da Internet no Brasil e da qual faz parte o Núcleo de Informação e Coordenação do Ponto BR (NIC.br), que tem a função de implementar as decisões e os projetos do comitê. Uma delas é o Registro.BR, plataforma onde são registrados todos os domínios brasileiros (CGI.br, 2021).

Este modelo de conexão onde a Embratel era a única empresa a distribuir a Internet para todos brasileiros se mostrou ineficiente e o mercado foi aberto a empresas privadas conhecidas como provedores que obtinham a conexão através da Embratel e vendiam aos clientes expandindo o número de clientes conectados à rede (Agência Brasil, 2021).

Com o ingresso desses novos usuários várias empresas privadas e órgãos governamentais passaram a oferecer serviços através da Internet como declaração de imposto de renda online, surgimento de e-mails gratuitos, surgimento de sites de busca, sites de jornais como o Jornal do Brasil Online, primeiro jornal brasileiro na Internet e programas para troca de mensagens fizeram e fazem sucesso até hoje (Kleina, 2018).

Paralelamente a evolução da Internet evolui-se também os dispositivos que a acessam, antes a grande maioria dos acessos eram feitos através de computadores desktop que hoje figuram como o segundo meio mais utilizado pelos brasileiros, perdendo a posição para os smartphones, outro meio de acesso em crescimento são as smart tvs, como podemos ver na figura 1 em pesquisa realizada pelo Instituto Brasileiro de Geografia e Estatística (IBGE, 2021).

Figura 1 - Tipos de equipamentos utilizados pelos brasileiros para acesso à Internet.

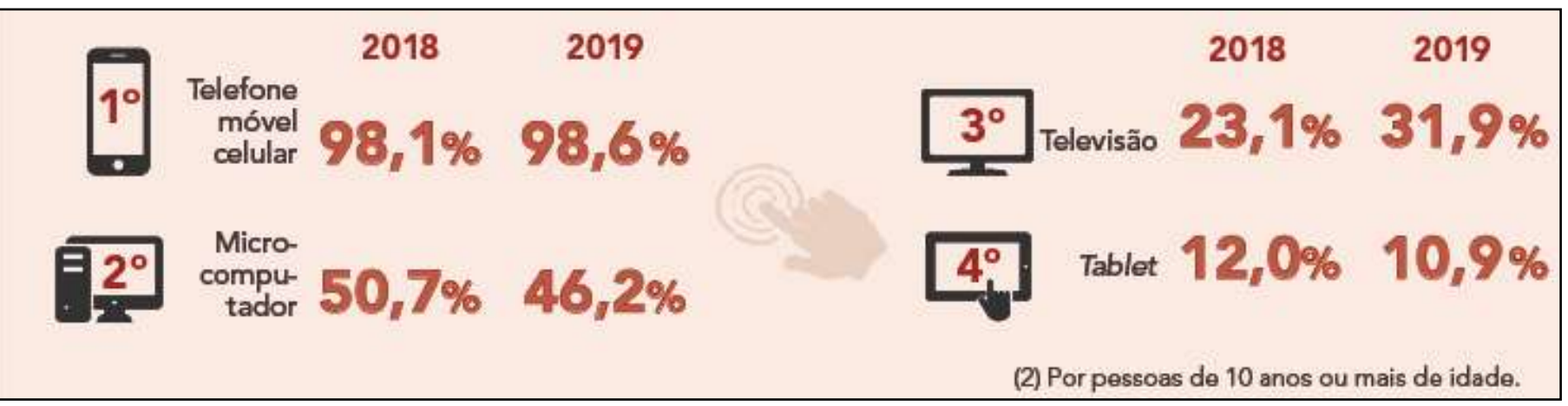

Fonte: IBGE (2021).

Se no início à Internet era restrita a troca de informações acadêmicas através de e-mails, grupos de notícias, transferências de arquivos e acesso remotos com o surgimento da Web surgiram novos serviços como sites atualizados em tempo real, redes sociais, blogs, conteúdos streaming, dispositivos inteligentes todos conectados entre si, trocando informações permitindo, por exemplo, através de um smartphone em qualquer lugar do mundo controlar dispositivos como câmeras de segurança, monitorar ambientes remotos com sensores inteligentes e até mesmo comandar robôs que realizam cirurgias em pacientes.

\subsection{Internet das coisas e a evolução das redes móveis de telefonia celular}

O termo IoT é uma tradução da língua inglesa da expressão Internet of Things (IoT), e nos traz a ideia de que a Internet pode estar presente em todas as coisas, possibilitando a fusão do mundo real e digital, ondes dispositivos conectados à Internet e em constante comunicação fornecem informações que facilitam a vida das pessoas no seu dia a dia. Ainda que este termo seja desconhecido para muitos, não é um conceito novo (Alecrim, 2019).

John Romkey foi o responsável pelo desenvolvimento do primeiro dispositivo IoT, apresentado em 1990 e 1991, 
durante a Conferência anual de Tecnologia da Informação, uma torradeira elétrica que podia ser ligada e desligada e um braço robótico que colocava a fatia de pão, ambos controlados via Internet (Postscapes, 2019).

O termo IoT foi criado somente em 1999 por Kevin Ashton - fundador do grupo de pesquisa Massachusetts Institute of Technology (MIT) Auto-ID Center atual Auto-ID Labs - durante a apresentação de um trabalho que buscava vincular o método de Radio-Frequency IDentification (RFID) a cadeia de suprimentos da Procter \& Gamble (P\&G). O objetivo de Ashton era que os computadores coletassem informações de objetos permitindo assim uma observação, identificação e compreensão do mundo pelos computadores sem as limitações humanas (Ashton, 2009).

Em uma entrevista a $18^{\mathrm{a}}$ edição da revista Inovação da Pauta, publicada pela Financiadora de Estudos e Projetos (Finep), Ashton (apud Rangel, 2015 p. 16) relata a importância da conversão entre as redes da comunicação humana e o mundo real das coisas:

Mas o que eu quis dizer à época, e ainda considero isso válido, se baseia na ideia de que estamos presenciando o momento em que duas redes distintas - a rede de comunicações humana (exemplificada na Internet) e o mundo real das coisas - precisam se encontrar. Um ponto de encontro onde não mais apenas "usaremos um computador", mas onde o "computador se use" independentemente, de modo a tornar a vida mais eficiente. Os objetos - as "coisas" estarão conectados entre si e em rede, de modo inteligente, e passarão a "sentir" o mundo ao redor e a interagir ("grifos do autor").

Um dos primeiros sistemas IoT utilizados pela indústria foi o Electronic Product Code (EPC) que é um sistema global de identificação baseado em RFID. Este sistema foi desenvolvido pelo grupo de pesquisa Auto-ID Labs e permitia controlar o estoque e rastrear os produtos na linha de produção, tudo isso através de tags que podem ser lidas a uma distância de até 9 metros, alcance muito maior que o código de barras por exemplo (GS1 Brasil, 2018) (Postscapes, 2019). Podemos citar a evolução da IoT com eventos que ocorreram e foram destaques dos anos 2000 em diante conforme mostra o Quadro 1.

Quadro 1 - Eventos destaques sobre IoT a partir do ano 2000

\begin{tabular}{|c|c|c|}
\hline AUTOR E ANO & NOME EVENTO & $\begin{array}{l}\text { ANO DO } \\
\text { EVENTO }\end{array}$ \\
\hline Postscapes (2019) & $\begin{array}{l}\text { A LG Corporation apresentou os primeiros planos de refrigeradores conectados à Internet, onde } \\
\text { podia-se ler } e \text {-mails, notícias, assistir televisão e sua principal função era determinar quando um } \\
\text { alimento deveria ser reabastecido. }\end{array}$ & 2000 \\
\hline Apple (2007) & Lançamento do primeiro smartphone da Apple. & 2007 \\
\hline Tecmundo (2017) & $\begin{array}{c}\text { Lançamento do primeiro smartphone com o Android, sistema operacional gratuito e atualmente o } \\
\text { mais utilizado. }\end{array}$ & 2008 \\
\hline Postscapes (2019) & $\begin{array}{l}\text { - Formada a Internet Protocol for Smart Objects (IPSO) - Organização sem fins lucrativos que } \\
\text { promovia a utilização do Protocolo IP em redes de objetos inteligentes. }\end{array}$ & 2008 \\
\hline Postscapes (2019) & $\begin{array}{l}\text { Primeiros termostatos inteligentes - Termostatos conectados via Wi-Fi que eram programáveis e } \\
\text { possuíam autoaprendizagem, otimizando o aquecimento e resfriamento de casas e empresas. }\end{array}$ & 2011 \\
\hline Techtudo (2015) & $\begin{array}{c}\text { Chegam as primeiras smart tvs ao Brasil - Os televisores agora contam com aplicativos de streaming } \\
\text { influenciando a diminuição de mídias físicas. }\end{array}$ & 2011 \\
\hline Apple (2014) & $\begin{array}{l}\text { Lançamento do primeiro smartwatch da Apple - Possibilita o rastreamento de exercícios físicos, era } \\
\text { equipado com recursos de saúde e permitia também os usuários armazenarem músicas e fotos. }\end{array}$ & 2015 \\
\hline $\begin{array}{l}\text { Sírio Libanês } \\
\quad(2016)\end{array}$ & $\begin{array}{c}\text { Realizada a Primeira Tele cirurgia no país - Primeira tele cirurgia do hemisfério sul é realizada no } \\
\text { hospital Sírio Libanez por um médico localizado nos Estados Unidos }\end{array}$ & 2016 \\
\hline $\begin{array}{l}\text { Agência Nacional } \\
\text { de } \\
\text { Telecomunicações } \\
\text { (2021) }\end{array}$ & $\begin{array}{c}\text { Agência Nacional de Telecomunicações aprova edital de leilão de 5G - Primeiros passos para a } \\
\text { instalação da nova tecnologia de redes móveis 5G, que impactará diretamente o funcionamento da } \\
\text { Internet das Coisas }\end{array}$ & 2021 \\
\hline
\end{tabular}

Fonte: Desenvolvido pelo autor a partir das referências contidas no quadro.

$\mathrm{O}$ interesse em IoT aumentou, surgindo constantemente em publicações no The Guardian, Scientific American $e$ Boston Globe, atingindo outro nível quando a UN's International Telecommunications Union (ITU) publicou seu primeiro 
relatório sobre o assunto em 2005 e desde o reconhecimento em 2008 é realizada a European IoT Conference, que reúne principais pesquisadores e profissionais da academia e da indústria para facilitar o compartilhamento de aplicações, resultados de pesquisa e conhecimento (International Conference for Industry and Academia, 2008).

A Figura 2 mostra a revolução na Internet móvel e como essa revolução mudou a forma de uso dos dispositivos móveis.

Figura 2 - Evolução da telefonia móvel.

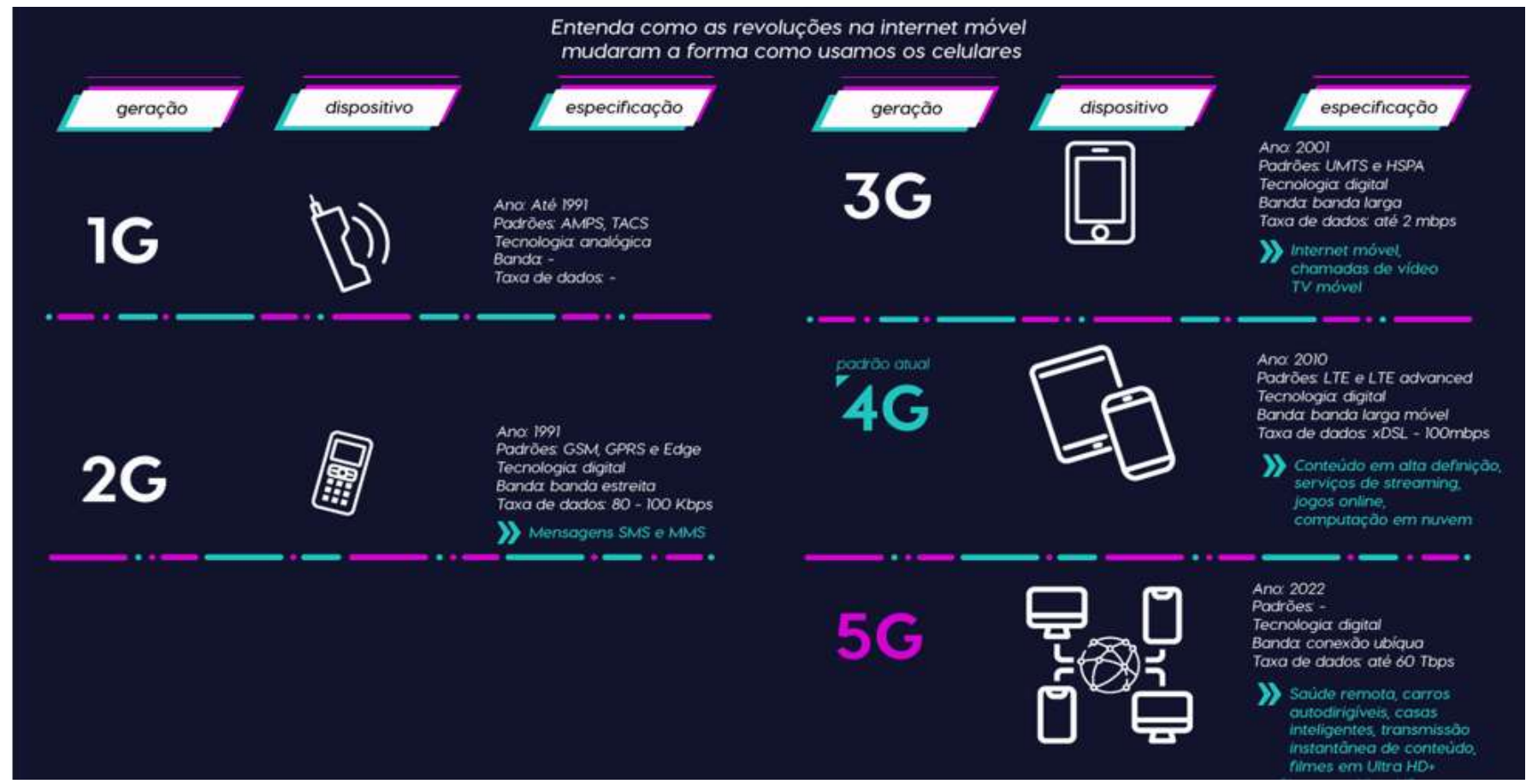

Fonte: FCC apud Agência Brasil (2021).

As tecnologias mais utilizadas e conhecidos para conectar dispositivos IoT são, NFC e Bluetooth ideais para dispositivos pessoais e comerciais que demandam baixas velocidades e alcance curto como smartwatches, cartões de crédito com chip, o Wi-Fi possui alcance e velocidades maiores e são utilizados em smart tvs e câmeras de segurança e por fim as redes móveis de telefonia celular, com uma cobertura maior, é muito utilizado por smartphones e dispositivos que estão localizados em locais remotos sem cobertura por outros meios de comunicação (Morais et al., 2018).

As redes móveis de telefonia celular são fundamentais para comunicação, possibilitando através de combinações de antenas a criação de várias células aumentando o sinal de cobertura e permitindo uma conexão ininterrupta conectando e transferindo o cliente a antena de melhor sinal, tudo de forma transparente. A evolução das redes móveis de telefonia celular foi classificada em gerações (Federal Communications Commission, 2021).

A primeira geração eliminou a necessidade de utilização de cabos para comunicação, permitindo somente chamadas analógicas de voz. Na segunda geração a rede analógica é substituída por uma rede digital, permitindo uma qualidade melhor nas ligações e a novidade é a implementação de envio e recebimento de mensagens de texto e multimídia (Federal Communications Commission, 2021).

$\mathrm{Na}$ terceira geração é habilitado o acesso à Internet móvel completo, acesso a sites, e-mails e também vídeo chamadas. Com velocidades de até 2 mbps foi classificada como a primeira geração de Internet móvel com banda larga de velocidade. A quarta geração com velocidades de até 100 mbps possibilita streaming de vídeos em alta resolução, jogos online vídeo conferência e outros recursos de alta velocidade (Agência Brasil, 2021). 
A quinta geração poderá oferecer em velocidades até 100 vezes mais rápidas que a geração anterior e com tempo de resposta quase zero permitirá a expansão da IoT ampliando a utilização de tecnologias inovadores como a telemedicina, onde um cirurgião poderá realizar um procedimento cirúrgico a distância com seus movimentos sendo executados instantaneamente por robôs, carros autônomos que utilizarão uma combinação de sensores conectados à Internet, realidade virtual, streaming de vídeos em ultra resolução etc. (Federal Communications Commission, 2021; Silva, \& et al., 2020)

\subsection{Perspectivas futuras e desafios para internet das coisas}

Nesta seção será discutido sobre perspectivas futuras de utilização da IoT e seus benefícios em mobilidade urbana, segurança e saúde pública, transportes e avanços na medicina; Desafios como infraestrutura para o funcionamento da IoT, segurança e privacidade deverão ser superados para sua utilização em larga escala.

\subsubsection{Cidades inteligentes}

A IoT com sua versatilidade auxiliará as cidades a solucionar suas demandas em diversas áreas como saúde, segurança, meio ambiente e trânsito, fornecendo informações relevantes e atuais possibilitando aos gestores tomadas de decisões claras e rápidas com intervenções específicas e até mesmo criação de políticas públicas (Oliveira, \& et al., 2021).

Atualmente os semáforos são controlados por tempo, medida que está se tornando defasada devido às constantes mudanças no modo de locomover que ocorrem nas cidades. Semáforos inteligentes analisarão o fluxo do trânsito e tomarão decisões em tempo real, otimizando a movimentação dos veículos (Brito, 2017).

Na segurança pública a IoT fornecerá dados que auxiliarão no combate e prevenção de crimes. Com análise de dados será possível acompanhar o índice de criminalidade de regiões e propor ações educativas, palestras, blitz como medidas de prevenção. O uso de dispositivos inteligentes ajudará em investigações e soluções de crimes, como exemplo o ShotSpotter, tecnologia que identifica através de sensores distribuídos a localização de um disparo de arma de fogo pelo som e comunica ao departamento policial responsável pela região (Brito, 2017).

\subsubsection{Internet das coisas no transporte}

As indústrias sempre investiram em pesquisas e desenvolvimento de novas tecnologias buscando aumentar a produtividade e competitividade. O investimento em sistemas autônomos mostra-se bastante promissor e tem dado os primeiros resultados promissores nas áreas de transporte com carros semi autônomos da fabricante Tesla, que permite o carro acelerar, frear e virar automaticamente, com supervisão ativa do motorista (Tesla, 2021).

O transporte rodoviário de cargas também é um setor que será influenciado por veículos autônomos, na China já existem testes com caminhões autônomos equipados com sensores Light Detection And Ranging (LiDAR), que conseguem detectar objetos a $1 \mathrm{~km}$ de distância, transportando cargas em rotas selecionadas (Tecmundo, 2021).

$\mathrm{Na}$ área de mineração a empresa Vale iniciará testes para que o transporte de minério de ferro realizado por caminhões fora de estrada, sejam operados de forma autônoma controlados por sistemas de computador, sistema de posicionamento global, radares e inteligência artificial, eles serão monitorados por salas de comandos a quilômetros de distância (Vale, 2019).

\subsubsection{Internet das coisas na saúde}

As pesquisas e desenvolvimentos de tecnologias voltadas para saúde tem avançado e muitas já refletem no nosso cotidiano, com soluções inteligentes que permitem mudar significativamente a rotina dos pacientes e profissionais da saúde. Com o auxílio de sensores e dispositivos vestíveis será possível identificar alterações como arritmia cardíaca, monitorar o desempenho cognitivo das pessoas e com este histórico identificar os primeiros sinais de doenças como Alzheimer e demência. 
Os dispositivos vestíveis atualmente somente coletam e informam ao usuário sobre sua saúde, cabendo ao usuário procurar uma ajuda médica. Futuramente esses dispositivos poderão estar ligados diretamente ao prontuário eletrônico e fornecer informações em tempo real auxiliando hospitais e clínicas médicas nas consultas e procedimentos (Moura, \& et al., 2020).

Com a integração entres os dispositivos e software pode-se identificar e rastrear instrumentos e materiais de alto valor agregado como órteses e próteses - que impactam no alto custo hospitalar - monitorar o funcionamento dos aparelhos hospitalares, prevenindo problemas e manutenções de última hora (Reis, 2018).

Cirurgias poderão ser realizadas remotamente e transmitidas utilizando a IoT, onde por exemplo em hospitais que não contam com especialistas naquele momento poderão realizar o procedimento com um profissional a distância controlando os equipamentos (Brito, 2017).

\subsubsection{Desafios para internet das coisas}

A quinta geração da Internet móvel será fundamental para a IoT pois permitirá o funcionamento de novos serviços que exigem um tempo de resposta ultrarrápido e demandam alta velocidade. Esta geração possui características superiores a geração anterior e também desafios que devem ser superados para o seu funcionamento como toda implementação de tecnologia nova (Lucca \& Mauro, 2020).

Utilizando ondas eletromagnéticas de frequências altas, possibilita um volume maior de transmissão de dados e também uma quantidade maior de dispositivos conectados simultaneamente. Frequências altas percorrem distâncias curtas e são sensíveis a obstáculos, desta forma são necessários mais equipamentos e antenas para propagação de sinais quando comparado a gerações anteriores, aumentando consideravelmente o custo da instalação pois para cada equipamento instalado é necessária uma infraestrutura física como construção de torres e energia elétrica (Lucca \& Mauro, 2020).

No Brasil a implementação da Internet de quinta geração ainda está na fase de licitação, a Agência Brasileira de Telecomunicações (ANATEL) divulgou a minuta do edital que contém os lotes das frequências ofertadas e as obrigações de cobertura. A previsão para o funcionamento da quinta geração de Internet móvel nas 27 capitais brasileiras é até julho de 2022, onde existirá a infraestrutura que possibilitará grandes mudanças tecnológicas com novos tipos de serviços e produtos (Agência Brasil, 2021).

Outros desafios que deverão ser superados para o funcionamento ideal da IoT é a segurança e privacidade das informações. Inicialmente a ideia de se conectar tudo é fascinante, mas os desafios de manter um sistema seguro são complexos e exigem investimentos em treinamento pessoal e equipamentos. Os dispositivos IoT geram uma grande quantidade de informações e essas informações devem ser armazenadas e o acesso deve ser restrito a quem realmente tem privilégio (Santos \& Sales, 2016).

Os primeiros ataques a sistemas integrados a IoT já começam a acontecer e seus efeitos causaram grandes prejuízos um deles teve como alvo o maior oleoduto dos Estados Unidos, responsável pelo transporte de $45 \%$ do diesel, gasolina e querosene de aviação de toda costa leste, obrigando o governo a declarar estado de emergência em algumas regiões do país (British Broadcasting Corporation, 2021).

\section{Considerações Finais}

Este artigo abordou de uma forma geral o surgimento e a evolução da Internet, desde a sua criação com objetivos militares e acadêmicos, sua expansão e abertura comercial na década de 1990 até os dias atuais. Posteriormente foi contextualizado o surgimento da Internet no Brasil, com as primeiras conexões internacionais entre universidades brasileiras e americanas até a abertura comercial permitindo a expansão da Internet em território brasileiro.

É retratado também que a usabilidade da Internet estava restrita apenas a uso de computadores, bem como os 
dispositivos de conexões com a rede mundial de computadores serviam para este fim, ou seja, somente utilizados em computadores de mesa. Essas reflexões nos fazem retornar ao problema de pesquisa - Como identificar o que de fato é a IoT e quais os desafios e perspectivas futuras a serem identificadas para a implementação desta tecnologia em larga escala? - Assim, em resposta a este problema destaca-se:

- A evolução dos dispositivos dos mais variados tipos integrados em smartphone, smart tv, smartwatch, expandindo ainda mais o acesso à Internet no período hodierno, nos fornece informações que auxiliam nas tomadas de decisões;

- Ademais, ocorreu a evolução das redes móveis de telefonia celular em especial a quinta geração, que com sua latência baixa permitirá a expansão da IoT;

- A IoT tem evoluído para cada vez mais fazer parte do cotidiano das pessoas, e dessa forma tem permitido não só automatizar operações que é importante, mas também uma convergência de IoT, entre a grande quantidade de dados coletados pelos diversos dispositivos, algoritmos e sistemas de aprendizados que juntos irão compor a Inteligência Artificial;

- No Brasil existe uma perspectiva da implementação das redes móveis de telefonia celular de quinta geração o que possibilitará a aplicabilidade destas tecnologias;

- A Segurança e a privacidade são temas que devem ser discutidos e implementados de maneira que o bem mais importante dessa era, que é a informação não seja acessada e manipulada por pessoas sem autorização. Conectividade, segurança e privacidade formam a base da IoT.

\section{Sugestões para trabalhos futuros:}

- Mesmo fazendo parte do nosso cotidiano nem todos tem conhecimento sobre seus conceitos, evolução, aplicabilidades e desafios. Assim sugere-se que sejam realizadas pesquisas para inserção das pessoas em ambientes que não usem a IoT e ambientes nos quais as decisões são tomadas com o auxílio da IoT, por exemplo pesquisas com grupos de controle;

- Como contribuição espera-se que este trabalho sirva como incentivo e possa nortear novos estudos, principalmente sobre a aplicabilidade da IoT na área social, como exemplo para entender qual o impacto da IoT na vida das pessoas atualmente, ou na área industrial para analisar como a IoT pode automatizar procedimentos tornando-os mais eficientes;

A partir do que foi exposto espera-se com esse artigo, que esta temática IoT tendo em vista a sua relevância, seja disseminada para o conhecimento de todos. Destarte, esses conceitos servirão como fundamentação para debates, questionamentos e reflexões.

\section{Referências}

Agência Brasil. (2021). Como era a Internet no Brasil antes da comercialização. Brasília. https://agenciabrasil.ebc.com.br/geral/noticia/2021-04/como-erainternet-no-brasil-antes-da-comercializacao

Agência Brasil. (2021). Leilão do 5G: entenda o que vem por aí e conheça as novidades. Brasília. https://agenciabrasil.ebc.com.br/geral/noticia/202104/leilao-do-5g-entenda-o-que-vem-por-ai-e-conheca-novidades

Agência Nacional de Telecomunicações. (2021). Anatel aprova edital de leilão de 5G. Brasília. https://www.gov.br/anatel/pt-br/assuntos/noticias/anatelaprova-o-edital-do-leilao-de-5g

Alecrim, E. (2019). O que é Internet das Coisas (IoT)?. São Paulo. https://www.infowester.com/iot.php

Apple. (2007). Apple Reinventa Telefone com o iPhone. São Paulo. https://www.apple.com/br/newsroom/2007/01/09Apple-Reinvents-the-Phone-with-iPhone

Apple. (2014). Apple Unveils Apple Watch_Apple’s Most Personal Device Ever. São Paulo. https://support.apple.com/kb/SP745?viewlocale=pt_BR\&locale= pt_BR

Ashton, K. (2009). That 'Internet of things' thing. RFiD Journal, 22(7):97-114.

Brasil. (2014) Lei $n^{\circ}$ 12.965, de 23 de abril de 2014. Estabelece princípios, garantias, direitos e deveres para o uso da Internet no Brasil. Brasília. http://www.planalto.gov.br/ccivil_03/_ato2011-2014/2014/lei/112965.htm 
British Broadcasting Corporation. (2021). O ataque de hackers a maior oleoduto dos EUA que fez governo declarar estado de emergência. São Paulo. https://www.bbc.com/portuguese/internacional-57055618

Brito, R. L. L. (2017). Potencial da Internet das Coisas na Saúde, Educação e Segurança Pública no Brasil. Recife.

Carvalho, T. C. M. B. (1997). Arquiteturas de redes de computadores OSI e TCP/IP. (2a ed.). Brisa.

CGI.br. (2021). História do CGI.br. São Paulo. https://www.cgi.br/historicos/\#1995

Embratel. (2020). Conheça a nossa história: Linha do Tempo. São Paulo. http://portal.embratel.com.br/embratel/55anos/

Federal Communications Commission. (2021). The Five G's - A Chronology. Washington. https://www.fcc.gov/5g-faqs

Gil, A. C. (2017). Como elaborar projetos de pesquisa. Atlas.

GS1 Brasil. (2018). EPC/RFID: entenda o que é e qual a importância para a sua indústria. Associação Brasileira de Automação. https://blog.gs1br.org/epcrfid-entenda-o-que-e/

International Conference for Industry and Academia. (2008). Internet of Things 2008. Zurich. https://iot-conference.org/iot2008

IBGE. (2021). Uso de Internet, televisão e celular no Brasil. Rio de Janeiro. https://educa.ibge.gov.br/jovens/materias-especiais/20787-uso-de-Internettelevisao-e-celular-no-brasil.html

Kleina, N. (2018). Como tudo começou: a história da Internet no Brasil. São Paulo. https://www.tecmundo.com.br/mercado/129792-tudo-comecou-historiaInternet-brasil-video.htm

Kurose, J. F. \& Ross, K. W. (2012). Computer Networking: A Top-Down Approach. (5a ed.). Pearson.

Lucca, J. \& Mauro, P. S G. (2020). Desafios da Tecnologia 5G. Catanduva.

Marconi, M. de A. (2017). Fundamentos de metodologia científica. Atlas.

Morais, I. S., \& et al. (2018). Introdução a big data e Internet das coisas (IOT). Porto Alegre.

Moura, F. R. E. ., Pitta, G. B. B. ., \& Tonholo, J. . (2020). Technological prospection of internet of things in the context of smart hospitals. Research, Society and Development, 9(11), e50791110081. https://doi.org/10.33448/rsd-v9i11.10081

Oliveira, M. (2011). Primórdios da rede: A história dos primeiros momentos da Internet no Brasil. Revista Fapesp, São Paulo, v. 180. https://revistapesquisa.fapesp.br/prim\%C3\%B3rdios-da-rede_/

Oliveira, P., \& et al. (2021). IoT nas Smart Cities: Revisão da literatura. Revista Ibérica de Sistemas e Tecnologias de Informação. Issue E42, pp.330-343.

Peterson, L. L., \& Davie, B. S. (2011). Computer Networks, Fifth Edition: A Systems Approach. San Francisco. Morgan Kaufmann Publishers Inc.

Postscapes. (2019). Internet of Things (IoT) History. https://www.postscapes.com/iot-history/

Rangel, R. (2015). Kevin Ashton - entrevista exclusiva com o criador do termo “Internet das Coisas". Revista Inovação da Pauta, Rio de Janeiro, ed. 18, p. 67. http://finep.gov.br/images/revista/revista18/index.html\#p=8

Reis, C. (2018). IoT na Saúde: o futuro já chegou. São Paulo. Revista Apólice. https://www.revistaapolice.com.br/2018/12/iot-na-saude-o-futuro-ja-chegou

Santos, B. P., \& et al. (2016). Internet das Coisas: da Teoria à Prática. Universidade Federal de Minas Gerais. Belo Horizonte

Santos, C. C. \& Sales, J. D. A. (2016). O Desafio da Privacidade na Internet das Coisas. Universidade Federal de Sergipe. Aracajú

Silva, L. G. P. da, Lemos, T. O., \& Rufino, H. L. P. (2020). The impact of the Internet of Things on education: a literature review. Research, Society and Development, 9(9), e710997770. https://doi.org/10.33448/rsd-v9i9.7770

Sírio Libanês. (2016). Brasil: É Realizada a Primeira Telecirurgia no país. São Paulo. https://www.hospitalsiriolibanes.org.br/imprensa/noticias/Paginas/Brasi 1-\%C3\%89-Realizada-a-Primeira-Telecirurgia-no-pa\%C3\%ADs-.aspx

Tanenbaum, A. S. (2011). Redes de Computadores. (5a ed.), São Paulo: Pearson Education.

Techtudo. (2015). TVs: relembre a história do eletrônico 'queridinho' dos brasileiros. Rio de Janeiro. https://www.techtudo.com.br/noticias/noticia/2015/06/ tvs-relembre-a-historia-do-eletronico-queridinho-dos-brasileiros.html

Tecmundo. (2017). A história da HTC, a primeira que lançou um smartphone Android. São Paulo. https://www.tecmundo.com.br/mercado/121585-historiahtc-primeira-lancou-smartphone-android-video.htm

Tecmundo. (2021). Startup coloca 5 mil caminhões autônomos nas estradas da China. São Paulo. https://www.tecmundo.com.br/mobilidade-urbana-smartcities/210375-startup-coloca-5-mil-caminhoes-autonomos-estradas-china.htm

Tesla. (2021). Future of Driving. Palo Alto. https://www.tesla.com/autopilot

Vale. (2019). Vale iniciará testes para implantação da operação autônoma em caminhões fora de estrada em Carajás. Rio de Janeiro. http://www.vale.com/brasil/PT/aboutvale/news/Paginas/vale-iniciara-testes-para-implantacao-da-operacao-autonoma-em-caminhoes-fora-de-estrada-emcarajas.aspx 